\title{
CORE EXISTENCE IN VERTICALLY DIFFERENTIATED MARKETS*
}

\author{
JEAN J. GABSZEWICZ, MARCO A. MARINI AND ORNELLA TAROLA
}

\begin{abstract}
We prove that a sufficient condition for the core existence in a $n$-firm vertically differentiated market is that the qualities of firms' products are equispaced along the quality spectrum. This result contributes to see that a fully collusive agreement among firms in such markets is more easily reachable when product qualities are not distributed too asymmetrically along the quality ladder.
\end{abstract}

Keywords: Vertically Differentiated Markets, Price Collusion, Core, Grand Coalition, Coalition Stability, Games with Externalities, Partition Function Games.

JEL Classification: D42, D43, L1, L12, L13, L41.

\section{Introduction}

The main aim of this note is to prove that a sufficient - albeit not necessary - condition for the core existence in a partition function game associated to a $n$-firm version of the classical vertically differentiated market (e.g., Mussa and Rosen 1978, Gabszewicz and Thisse $1979,1980)$ is that the qualities of products sold by the firms are equispaced along the quality spectrum. In addition, we show that, when this regularity condition is relaxed, the core can be easily empty.

There exist very few contributions dealing with the existence of core in oligopoly games with heterogeneous firms. ${ }^{1}$ Our result contributes to see that a fully collusive agreement among firms in such markets is more easily reachable when the product qualities are not distributed too asymmetrically along the quality ladder.

Given that the vertical differentiated market is a setting with strategic interdependence, the most appropriate coalitional game derived from it is a game in partition function (Thrall

Date: September 2016.

Jean J. Gabszewicz, CORE, Université Catholique de Louvain, Belgium. E-mail: jean.gabszewicz@uclouvain.be.

Marco A. Marini, Department of Social and Economic Sciences, Sapienza Università di Roma, Italy and CREI. Address: Piazzale Aldo Moro, 5, 00184, Roma.(Italy). Tel. +39-06-77274044. E-mail: marini@dis.uniroma1.it (corresponding author).

Ornella Tarola, Department of Social and Economic Sciences, Università di Roma La Sapienza, Italy. E-mail: ornella.tarola@uniroma1.it.

*We are grateful to the editor Roberto Serrano, to two anonymous reviewers and to Rabah Amir, Sergio Currarini, Sébastien Mitraille and to the participants in Oligo Workshop 2016 in Paris Dauphine and in EARIE 2016 Conference in Nova Universidade de Lisbon for their useful comments and suggestions.

${ }^{1}$ Zhao (2013) examines the existence of $\alpha$-, $\gamma$ - and $\delta$-core in a three-firm linear Cournot oligopoly with different marginal costs. In a differentiated quantity oligopoly with three (or four firms) Watanabe and Matsubayashi (2013) show that for any degree of product differentiation the $\gamma$-core is nonempty while the $\delta$-core only exists in presence of high product differentiation. For a more detailed account of the works dealing with coalitional agreements in oligopoly games, see Marini 2009 and 2016 and Currarini and Marini 2015 . 
and Lucas 1963). This in line with the recent interest in coalitional games with externalities (see, e.g., Maskin 2003, Ray 2007, Hafalir 2007, de Clippel and Serrano 2008, Bloch and van den Nouweland 2014, Ray and Vohra 2015). It is well known that, when externalities are at work across coalitions, the use of a coalitional worth requires some assumptions on the expected behaviour of players outside every deviating coalition. In such cases, core allocations may fail to exist even in convex games, for instance when players in the complementary coalition are expected to remain together, as in the delta core (Hart and Kurz 1983), also denoted projection core in the recent axiomatization by Bloch and van den Nouweland 2014. Moreover, since in the case of vertically differentiated markets the coalitional worth possesses positive coalition externalities, ${ }^{2}$ the delta or projection-core is the smallest core and, therefore, its existence implies the existence of all other possible versions of core in games with simultaneous moves. In this paper, we use this notion of core to provide the strongest existence result for the class of games considered here.

\section{Vertically Differentiated Market}

Let $n$ firms $i=1,2, \ldots, n$ offer $n$ quality variants $q_{1}, q_{2}, \ldots, q_{n}$, respectively, with $q_{i} \in(0, \infty)$ and $q_{n}>q_{n-1}>\ldots>q_{1}$ to a population of consumers. As in Mussa and Rosen (1978) consumers are indexed by $\theta$ and uniformly distributed in the interval $[0, \beta]$, with $\beta<\infty$. As usual, the parameter $\theta$ captures consumers' willingness to pay for quality. Each consumer can either buy one unit of a variant or not buying at all. Formally, consumer's utility is given by

$$
U(\theta)=\left\{\begin{array}{c}
\theta q_{i}-p_{i} \text { when buying variant } i \\
0 \text { when not buying, }
\end{array}\right.
$$

where $p_{i} \in[0, \bar{p}]$ with $0<\bar{p}<\infty$ is the price charged by firm $i$ for its variant $q_{i}$. From the above formulation, the marginal consumer buying variant $i=1$ is

$$
\theta_{1}=\frac{p_{1}}{q_{1}}
$$

and the market is uncovered, with some consumers excluded from buying even the bottomquality variant. In general, the consumer indifferent between buying variant $i-1$ and $i$ is, for $i=2,3, \ldots, n$

$$
\theta_{i}=\frac{p_{i}-p_{i-1}}{q_{i}-q_{i-1}}
$$

with $p_{i}>p_{i-1}$. When considering price competition, the payoffs of all firms can be easily characterized by the payoff of three types of firms in the quality spectrum: (i) top quality (ii) intermediate quality and (iii) bottom quality firm. Since in the model product qualities are exogenously given, we disregard costs to simplify calculations. ${ }^{3}$ The top quality firm (denoted $i=n$ ) sets a price $p_{n}$ maximizing its profit

$$
\Pi_{n}=\left(\beta-\frac{p_{n}-p_{n-1}}{q_{n}-q_{n-1}}\right) p_{n},
$$

\footnotetext{
${ }^{2}$ This means that every firm is advantaged when rivals merge in coalitions.

${ }^{3}$ It can be shown that the presence of quality-dependent fixed costs does not change the nature of the results obtained here.
} 
whereas every intermediate firm $i=2,3, \ldots, n-1$ selects a price $p_{i}$ to maximize

$$
\Pi_{i}=\left(\frac{p_{i+1}-p_{i}}{q_{i+1}-q_{i}}-\frac{p_{i}-p_{i-1}}{q_{i}-q_{i-1}}\right) p_{i} .
$$

Finally, the bottom quality firm $(i=1)$, sets a price $p_{1}$ to maximize

$$
\Pi_{1}=\left(\frac{p_{2}-p_{1}}{q_{2}-q_{1}}-\frac{p_{1}}{q_{1}}\right) p_{1} .
$$

Note that, from (2.2)-(2.4), firms' profit functions are continuous and concave in their own prices. Moreover, firms' choice sets are compact and convex and best-replies are contractions, ${ }^{4}$ so the existence of a unique (noncooperative) Nash equilibrium $n$-price vector p* associated to the $n$ variants $\left(q_{1}, q_{2}, \ldots, q_{n}\right)$ is guaranteed for any (finite) number of firms competing in the market. ${ }^{5}$ Moreover, the optimal reply of every firm is given by

$$
p_{n}\left(p_{n-1}\right)=\gamma p_{n-1}+\frac{\beta}{2}\left(q_{n}-q_{n-1}\right)
$$

for the top-quality firm $(i=n)$

$$
p_{i}\left(p_{i-1}, p_{+1}\right)=\frac{\gamma p_{i-1}\left(q_{i+1}-q_{i}\right)+\lambda p_{i+1}\left(q_{i}-q_{i-1}\right)}{\left(q_{i+1}-q_{i-1}\right)},
$$

for all intermediate firms $i=2,3, \ldots,(n-1)$ and

$$
p_{1}\left(p_{2}\right)=\gamma \frac{q_{1}}{q_{2}} p_{2}
$$

for the bottom-quality firm $i=1$, where $\gamma=\lambda=1 / 2$ at the noncooperative equilibrium, $\gamma=\lambda=1$ both under full collusion and when a firm lies inside a coalition of firms, and $\gamma=1 / 2$ and $\lambda=1$ (or $\gamma=1$ and $\lambda=1 / 2$ ) when a firm competes with its left (right) neighbour and colludes with its right (left) neighbour. This implies that every firm benefits from rivals' cartelisation and the coalitional worth (joint profit) of firms exhibits positive coalitional externalities: from (2.6)-(2.8) it ensues that all firms' optimal replies are positively sloped and their slope increases with (partial or full) collusion. Thus, rivals' cartelisation increases all firms' prices and, hence, their payoffs.

2.1. Grand Coalition Payoff. When all firms form a cartel they maximize the sum of firms' payoffs. As shown in Gabszewicz et al. (2016), under full price collusion all firms set prices $p_{i}^{c}$ such that their market shares are nil for all firms but the top-quality one $(i=n)$. This is is easy to show. Using (2.6)-(2.8) with $\gamma=\lambda=1$ for all firms, the following price is obtained

$$
p_{i}^{c}=\frac{1}{2} \beta \sum_{j \leq i} \delta_{j}
$$

\footnotetext{
${ }^{4}$ A sufficient condition for the contraction property to hold is (see, for instance, Vives 2000, p.47):

$$
\frac{\partial^{2} \Pi_{i}}{\partial\left(p_{i}\right)^{2}}+\sum_{j \neq i}\left|\frac{\partial^{2} \Pi_{i}}{\partial p_{i} \partial p_{j}}\right|<0
$$
}

which, using (2.3) for all intermediate firms $i=2, \ldots, n-1$, becomes

$$
-\frac{2\left(q_{i+1}-q_{i-1}\right)}{\left(q_{i+1}-q_{i}\right)\left(q_{i}-q_{i-1}\right)}+\frac{q_{i+1}-q_{i-1}}{\left(q_{i+1}-q_{i}\right)\left(q_{i}-q_{i-1}\right)}=\frac{q_{i-1}-q_{i+1}}{\left(q_{i+1}-q_{i}\right)\left(q_{i}-q_{i-1}\right)}<0
$$

which is respected for $q_{n}>q_{n-1}>\ldots>q_{1}$. The same applies for top and bottom quality firms.

${ }^{5}$ See, for instance Friedman (1991), p.84. 
where $\delta_{j}=\left(q_{j}-q_{j-1}\right)$ is the quality gap of every firm $j$ selling goods of lower or equal quality than firm $i$, and $\delta_{1}=\left(q_{1}-q_{0}\right)=q_{1}$. Inserting (2.9) in every firm's market share $D_{i}$, we obtain:

$$
D_{1}\left(p_{1}^{c}, p_{2}^{c}\right)=\left(\frac{p_{2}^{c}-p_{1}^{c}}{\delta_{2}}-\frac{p_{1}^{c}}{\delta_{1}}\right)=\left(\frac{\frac{1}{2} \beta\left(\delta_{1}+\delta_{2}\right)-\frac{1}{2} \beta \delta_{1}}{\delta_{2}}-\frac{\frac{1}{2} \beta \delta_{1}}{\delta_{1}}\right)=0
$$

for the bottom quality firm,

$$
\begin{aligned}
D_{i}\left(p_{i-1}^{c}, p_{i}^{c}, p_{i+1}^{c}\right) & =\left(\frac{p_{i+1}^{c}-p_{i}^{c}}{\delta_{i+1}}-\frac{p_{i}^{c}-p_{i-1}^{c}}{\delta_{i-1}}\right)= \\
& =\left(\frac{\frac{1}{2} \beta \sum_{j \leq i+1} \delta_{j}-\frac{1}{2} \beta \sum_{j \leq i} \delta_{j}}{\delta_{i+1}}-\frac{\frac{1}{2} \beta \sum_{j \leq i} \delta_{j}-\frac{1}{2} \beta \sum_{j \leq i-1} \delta_{j}}{\delta_{i-1}}\right)= \\
& =\left(\frac{\frac{1}{2} \beta \delta_{i+1}}{\delta_{i+1}}-\frac{\frac{1}{2} \beta \delta_{i}}{\delta_{i}}\right)=0 .
\end{aligned}
$$

for any intermediate quality firm, and

$$
\begin{aligned}
D_{n}\left(p_{n-1}^{c}, p_{n}^{c}\right) & =\left(\beta-\frac{p_{n}^{c}-p_{n-1}^{c}}{q_{n}-q_{n-1}}\right)=\left(\beta-\frac{\frac{1}{2} \beta \sum_{j \leq n} \delta_{j}-\frac{1}{2} \beta \sum_{j \leq n-1} \delta_{j}}{\delta_{n}}\right)= \\
& =\left(\beta-\frac{\frac{1}{2} \beta \delta_{n}}{\delta_{n}}\right)=\frac{1}{2} \beta,
\end{aligned}
$$

for the top quality firm. Thus, when colluding together all firms cover only half of the market and the grand coalition payoff is:

$$
v(N)=\sum_{i \in N} \Pi_{i}^{\{N\}}=\sum_{i=1}^{n} p_{i} D_{i}=\frac{1}{4} \beta^{2} q_{n} .
$$

2.2. Coalitional Payoffs. The $n$ firms can also collude organizing themselves in partition $P=\left(S_{1}, S_{2}, \ldots, S_{m}\right)$ different from the grand coalition. Every firm can actively collude in prices only with its left (lower quality), with its right (higher quality) or with both its closest competitors by forming bottom, intermediate or top quality cartels. ${ }^{6}$

Definition 1. (i) A bottom cartel $S_{B} \subset N$ is a coalition formed by consecutive intermediate firms $i=2, \ldots, n-1$ also including the bottom quality firm $i=1$. (ii) An intermediate cartel $S_{I} \subset N$ is a coalition only formed by consecutive intermediate firms $i=2, \ldots, n-1$. (iii) $A$ top cartel $S_{T} \subset N$ is a coalition formed by consecutive intermediate firms $i=2, \ldots, n-1$, also including the top quality firm $i=n$.

In the next proposition, we characterize the variants produced by the firms belonging to: (i) an intermediate cartel; (ii) a bottom cartel; (iii) a top cartel.

Proposition 1. (i) A bottom cartel only produces in equilibrium the top quality variant among those formerly produced by its firms. (ii) Any intermediate cartel only produces in equilibrium the top and the bottom quality variants among those formerly produced by its firms. (iii) Any top cartel only produces in equilibrium the top and the bottom quality variants among those formerly produced by its firms.

Proof. See Gabszewicz et al. (2016).

\footnotetext{
${ }^{6}$ Without forming cartels among consecutive firms, i.e producing adjacent variants firms' collusion does not affect price behaviour.
} 
Proposition 1 enables to characterize the number of variants marketed by the firms in any feasible partition $P=\left(S_{1}, S_{2}, \ldots, S_{m}\right)$ for $m \leq n$ and will be used extensively to prove the main paper result.

\section{Core stability}

This section analyses the stability of full price collusion, i.e. the situation in which all firms in the industry collude in prices. In particular, the next proposition shows that, when all firms' quality variants are equispaced (i.e. spaced at equal distance), it is always possible to find a division of the monopoly profit which makes the whole industry cartel stable against individual or coalitional deviations by firms.

We can formally associate to the described vertically differentiated market a partition function game $G=(N, v(S ; P))$, where $N$ is the set of firms and $v(S ; P): 2^{N} \times \mathcal{P} \rightarrow \mathcal{R}$ is the worth associated to every coalition of firms $S \subset N$ embedded in a partition $P \in \mathcal{P}$, where $\mathcal{P}$ is the set of all feasible partitions of the $N$ firms. We can now define the core of a partition function game.

Definition 2. A vector of payoffs $x=\left(x_{1}, x_{2}, \ldots, x_{n}\right)$ with $\sum_{i \in N} x_{i}=v(N)$ is in the core of the partition function game $G$ if, for every $S \subset N$ and every partition $P$ in which $S$ can be embedded, $\sum_{i \in S} x_{i} \geq v(S ; P)$.

We are now ready to prove our main result:

Proposition 2. Let market variants $q_{1}, q_{2}, \ldots, q_{n}$ be equispaced with $\left(q_{i}-q_{i-1}\right)=\delta \in(0, \infty)$ for every $i=1,2, \ldots, n$, and $q_{0}=0$. Then, the core of the partition function game $G$ associated to the $n$-firm vertically differentiated market is nonempty.

Proof. In our model of vertical differentiation, when a coalition of firms $S \subset N$ forms, its maximal coalitional payoff is obtained when the remaining firms in $N-S$ stick together in the complementary coalition $\{N-S\}$. Therefore, if the core is nonempty when the coalitional worth $v(S ; P)$ is computed for $P=\{S, N-S\}$, it will a fortiori be nonempty under any other partition $P \in \mathcal{P}$ in which $S$ can be embedded. For this reason, in what follows, we only need to prove that there exists an allocation $\mathbf{x}=\left(x_{1}, x_{2}, \ldots, x_{n}\right)$ of the grand coalition payoff $v(N)$ such that, for all $S \subset N, \sum_{i \in S} x_{i} \geq v(S ;\{S, N-S\})$. In particular, we prove this result by constructing a specific allocation respecting this requirement. Since the payoff obtained by every firm $i$ in partition $P=\{i, N-i\}$ is crucial to build such allocation, let us start from it. We consider first the payoff of the top quality firm (denoted $i=n$ ), in partition $P=\{n, N-n\}$. In this case, by Proposition 1, only two variants remain on sale, $q_{n}$ from firm $n$ and $q_{n-1}$ from the remaining firms merged in the bottom cartel $S_{B}=\{N-n\}$. As a result, in the new equilibrium under equispaced variants ${ }^{7}$

$$
v(n ;\{n, N-n\})=\Pi_{n}^{\{n, N-n\}}=\frac{4 \beta^{2} q_{n}^{2}\left(q_{n}-q_{n-1}\right)}{\left(4 q_{n}-q_{n-1}\right)^{2}}=\frac{4 \beta^{2} \delta n^{2}}{(3 n+1)^{2}} .
$$

As a second step, let us consider the payoff of the bottom-quality firm in partition $P=$ $\{1, N-1\}$. By Proposition 1, in this case only three variants remain on sale, $q_{1}, q_{2}$ and $q_{n}$, where $q_{2}$ and $q_{n}$ are offered by the firms merged in the top cartel $S_{T}=\{N-1\}$. In this new

\footnotetext{
${ }^{7}$ That is, for $\left(q_{1}-q_{0}\right)=\left(q_{2}-q_{1}\right)=. .=\left(q_{n}-q_{n-1}\right)=\delta$, and, hence, $q_{1}=\delta, q_{2}=2 \delta, \ldots, q_{n}=n \delta$.
} 
equilibrium, the payoff obtained by firm $i=1$ is

$$
v(1 ;\{1, N-1\})=\Pi_{1}^{\{1, N-1\}}=\frac{\beta^{2} q_{1} q_{2}\left(q_{2}-q_{1}\right)}{\left(4 q_{2}-q_{1}\right)^{2}}=\frac{2 \beta^{2} \delta}{49} .
$$

Finally, let us consider the payoff obtained by every intermediate firm $i=2, \ldots, n-1$ in partition $P=\left\{S_{B}, i, S_{T}\right\}$, where $S_{B}$ and $S_{T}$ are the bottom and top cartel neighbouring firm $i$. In this case, at most four variants remain on sale, namely $q_{i-1}$ from $S_{B}, q_{i}$ from $i$ and $q_{i+1}$ and $q_{n}$ from $S_{T}$, yielding:

$$
v(i ;\{i, N-i\})=\Pi_{i}^{\{i, N-i\}}=\frac{q_{i}^{2} \beta^{2}\left(q_{i}-q_{i-1}\right)\left(q_{i+1}-q_{i}\right)\left(q_{i+1}-q_{i-1}\right)}{\left(2 q_{i-1} q_{i}+q_{i-1} q_{i+1}-4 q_{i} q_{i+1}+q_{i}^{2}\right)^{2}}=\frac{\delta \beta^{2}(i)^{2}}{(6 i+1)^{2}} .
$$

Now, using (2.10) and (3.1)-(3.3) it is easy to see that, under equispaced variants, inequality

$$
v(N) \geq v(1 ;\{1, N-1\})+\sum_{i=2}^{n-1} v(i,\{i, N-i\})+v(n ;\{n, N-n\}),
$$

writes as

$$
\frac{n}{4} \geq \frac{2}{49}+\sum_{i=2}^{n-1} \frac{(i)^{2}}{(6 i+1)^{2}}+\frac{4 n^{2}}{(3 n+1)^{2}}
$$

and the latter expression holds with strict inequality for any number of firms $n \geq 2$.

Let us now construct a specific allocation $\widehat{x}=\left(\widehat{x}_{1}, \widehat{x}_{2}, \ldots, \widehat{x}_{n}\right)$ assigning to every firm $i=$ $1,2, \ldots, n$ a share $s_{i}$ of the grand coalition payoff $v(N)$ equal to

$$
s_{i}=\frac{v(i ;\{i, N-i\})}{\sum_{i \in N} v(i ;\{i, N-i\})},
$$

such that $\sum_{i \in N} s_{i}=1$, that is

$$
\widehat{x}=\left(s_{1} v(N), s_{2} v(N), \ldots, s_{n} v(N)\right) .
$$

Thus, since (3.4) holds with strict inequality, it ensues that for every firm $i=1,2, \ldots, n$

$$
\widehat{x}_{i}=s_{i} v(N)>s_{i} \sum_{i=1}^{n} v(i ;\{i, N-i\})=v(i ;\{i, N-i\}),
$$

implying that the selected allocation $\widehat{x} \in \mathcal{R}^{n}$ is robust against any individual firm's deviations.

As a second step, we need to look at the payoff obtained by any feasible coalition of firms. Let us assume again that every forming coalition expects the remaining firms to stick together in the complementary coalition (delta or projection expectations). As a result, when a coalition of firms in a bottom cartel $S_{B} \subset N$ forms under partition $P=\left\{S_{B}, N-S_{B}\right\}$, by Proposition 1 only variants $q_{h}, q_{h+1}$ and $q_{n}$ remain on sale $\left(q_{h}\right.$ from $S_{B}$ and $q_{h+1}$ and $q_{n}$ from $\left\{N-S_{B}\right\}$ ), where $q_{h}$ denotes the highest quality variant in $S_{B}$. The worth of any bottom cartel $S_{B}$ is, therefore,

$$
v\left(S_{B} ;\left\{S_{B}, N-S_{B}\right\}\right)=\sum_{i \in S_{B}} \Pi_{i}=\Pi_{h=\max \{i\}_{i \in S_{B}}}=\frac{\beta^{2} q_{h} q_{h+1}\left(q_{h+1}-q_{h}\right)}{\left(4 q_{h+1}-q_{h}\right)^{2}}=\frac{\delta \beta^{2} h(h+1)}{(3 h+4)^{2}} .
$$

From (3.5) and (3.6), for every $S_{B} \subset N$ inequality

$$
\sum_{i \in S_{B}} \widehat{x}_{i} \geq v\left(S_{B} ;\left\{S_{B}, N-S_{B}\right\}\right)
$$


writes, under equispaced variants, as

$$
\frac{\frac{2}{49}+\sum_{i=2}^{h} \frac{(i)^{2}}{(6 i+1)^{2}}}{\frac{2}{49}+\sum_{i=2}^{n-1} \frac{(i)^{2}}{(6 i+1)^{2}}+\frac{4 n^{2}}{(3 n+1)^{2}}} \frac{h(h+1)}{(3 h+4)^{2}}
$$

and (3.7) holds with strict inequality for every number of firms $n$ and for every $h=2, \ldots n-1$. Expression (3.7) ensures that no bottom cartel $S_{B}$ can improve upon $\sum_{i \in S_{B}} \widehat{x}_{i}$, the joint payoff assigned by allocation $\widehat{x} \in \mathcal{R}^{n}$ to members of $S_{B}$.

When, in turn, a top cartel $S_{T} \subset N$ forms under partition $P=\left\{S_{T}, N-S_{T}\right\}$, only three variants remain on sale, $q_{l-1}$ from $N-S_{T}$ and $q_{l}$ and $q_{n}$ from $S_{T}$, for $l$ denoting the lowest quality firm in $S_{T}$. Hence, under equispaced variants,

$$
\begin{aligned}
v\left(S_{T} ;\left\{S_{T}, N-S_{T}\right\}\right) & =\sum_{i \in S_{T}} \Pi_{i}^{\left\{S_{T}, N-S_{T}\right\}}=\Pi_{l=\min \{i\}_{i \in S_{T}}}+\Pi_{n}= \\
& =\frac{\beta^{2} q_{l} q_{l-1}\left(q_{l}-q_{l-1}\right)}{\left(4 q_{l}-q_{l-1}\right)^{2}}+\frac{1}{4} \frac{\beta^{2}\left(4 q_{l} q_{n}-q_{l-1} q_{n}-3 q_{l-1} q_{l}\right)}{\left(4 q_{l}-q_{l-1}\right)}= \\
& =\frac{\delta \beta^{2} l(l+1)}{(3 l+4)^{2}}+\frac{1}{4} \frac{\delta \beta^{2}\left(3 l+n+3 l \cdot n-3 l^{2}\right)}{(3 l+1)} .
\end{aligned}
$$

Note that this expression is decreasing in $l$, since the highest $l$ the smaller is the size of the top cartel $S_{T}$. Now, for every $S_{T} \subset N$,

$$
\sum_{i \in S_{T}} \widehat{x}_{i} \geq v\left(S_{T} ;\left\{S_{T}, N-S_{T}\right\}\right)
$$

under equispaced variants corresponds to

$$
\frac{\sum_{i=l}^{n-1} \frac{(i)^{2}}{(6 i+1)^{2}}+\frac{4 n^{2}}{(3 n+1)^{2}}}{\frac{2}{49}+\sum_{i=2}^{n-1} \frac{(i)^{2}}{(6 i+1)^{2}}+\frac{4 n^{2}}{(3 n+1)^{2}}} \frac{l(l+1)}{(3 l+4)^{2}}+\frac{1}{4} \frac{\left(3 l+n+3 l \cdot n-3 l^{2}\right)}{(3 l+1)},
$$

which holds with strict inequality for every number of firms $n$ and every $l=2, \ldots n-1$. Finally, when an intermediate cartel $S_{I} \subset N$ forms under partition $P=\left\{S_{B}, S_{I}, S_{T}\right\}$, by Proposition 1 at most five variants remain on sale: $q_{l-1}$ from $S_{B}, q_{l}$ and $q_{h}$ from $S_{I}$, and $q_{h+1}$ and $q_{n}$ from $S_{T}$, where, in turn, $l$ and $h$ stands for the lowest and highest quality firms in cartel $S_{I}$. The payoff obtainable by an intermediate cartel is, therefore,

$$
\begin{gathered}
v\left(S_{I} ;\left\{S_{B}, S_{I}, S_{T}\right\}\right)=\Pi_{S_{I}}^{\left\{S_{B}, S_{I}, S_{T}\right\}}=\frac{4 \beta^{2} q_{l-1} q_{l}\left(q_{l}-q_{l-1}\right)\left(q_{h+1}-q_{h}\right)^{2}}{\left(q_{l-1} q_{h}-9 q_{l-1} q_{l}-4 q_{l-1} q_{h+1}-4 q_{l} q_{h}+16 q_{l} q_{h+1}\right)^{2}}+ \\
+\frac{\beta^{2}\left(q_{h+1}-q_{h}\right)\left(4 q_{l} q_{h+1}-3 q_{l-1} q_{l}-q_{l-1} q_{h+1}\right)\left(4 q_{l} q_{h}-q_{l-1} q_{h}-3 q_{l-1} q_{l}\right)}{\left(q_{l-1} q_{h}-9 q_{l-1} q_{l}-4 q_{l-1} q_{h+1}-4 q_{l} q_{h}+16 q_{l} q_{h+1}\right)^{2}},
\end{gathered}
$$

that, under equispaced variants, can be written as

$$
\Pi_{S_{I}}^{\left\{S_{B}, S_{I}, S_{T}\right\}}=\frac{1}{16} \frac{\delta \beta^{2}\left(3 l^{2}-6 l-3 h l-h-1\right)\left(3 l^{2}-3 l-3 h l-h\right)}{\left(h+5 l+2 h l-2 l^{2}+1\right)^{2}}+\frac{\delta \beta^{2}(l-1) l}{4\left(3 h+21 l+9 h l-9 l^{2}+4\right)^{2}} .
$$


Thus, for every $S_{I} \subset N$

$$
\sum_{i \in S_{I}} \widehat{x}_{i} \geq v\left(S_{I} ;\left\{S_{I}, N-S_{I}\right\}\right)
$$

is

$$
\frac{\left(\sum_{i=l}^{h} \frac{(i)^{2}}{(6 i+1)^{2}}\right)}{\frac{2}{49}+\sum_{i=2}^{n-1} \frac{(i)^{2}}{(6 i+1)^{2}}+\frac{4 n^{2}}{(3 n+1)^{2}}} \frac{n}{4} \geq \frac{1}{16} \frac{\left(3 l^{2}-6 l-3 h l-h-1\right)\left(3 l^{2}-3 l-3 h l-h\right)}{\left(h+5 l+2 h l-2 l^{2}+1\right)^{2}}+\frac{(l-1) l}{4\left(3 h+21 l+9 h l-9 l^{2}+4\right)^{2}},
$$

which, again, holds for any number of firms $n$ and for any $l=2, \ldots n-2$ and $h=3, \ldots, n-1$, with $l<h$. As a result, the selected allocation $\widehat{x}$ distributes the grand coalition payoff in a way that no coalition of firms $S \subset N$ can, by leaving the grand coalition $N$, obtain a better payoff. The core is, therefore, nonempty.

3.1. Endogenous Qualities. It can be shown that, when $N=\{1,2,3\}$, the core is nonempty also when firms are allowed to select endogenously both qualities and prices. Following Gabszewicz et al. (2015), the grand coalition sets endogenously a product quality $q^{\{N\}}=0.25$ and, hence, $v(N)=0.03125 \beta$, which is sufficient to prevent individual deviations, given that: $v(N)=0.03125 \beta>v(1 ;\{1,23\})+v(2 ;\{2,13\})+v(3 ;\{12,3\})=0.00152 \beta+0.00152 \beta+0.02443 \beta$. Moreover, there exist allocations $x=\left(x_{1}, x_{2}, x_{3}\right)$ distributing $v(N)$ in such a way that no coalition $S \subset N$, by selecting its optimal quality and price, has an incentive to deviate. Using our sharing rule $s=\left(s_{1}, s_{2}, s_{3}\right)=(0.0533,0.0533,0.8893)$, we obtain that

$$
\begin{aligned}
& \sum_{i \in\{1,2\}} \widehat{x}_{i}=0.0033 \beta>v(12 ;\{12,3\})=0.00152 \beta, \\
& \sum_{i \in\{1,3\}} \widehat{x}_{i}=0.0945 \beta>v(13 ;\{13,2\})=0.02443 \beta, \\
& \sum_{i \in\{2,3\}} \widehat{x}_{i}=0.0945 \beta>v(23 ;\{1,23\})=0.02443 \beta,
\end{aligned}
$$

and the core is, therefore, nonempty. However, it can be shown that, with only three firms, the nonemptiness of core always holds for any distribution of product qualities. For core emptiness to arise, the presence in the market of at least four firms are required, as the next example will show.

3.2. An Empty Core Example. Let us consider the case of four firms selling four different variants $q_{1}, q_{2}, q_{3}$ and $q_{4}$. In this case, if the top cartel $S_{T}=\{234\}$ decides to leave the grand coalition $\{N\}$ and partition $P=\{1,234\}$ forms, it gains:

$$
v((234) ;\{1,234\})=\Pi_{234}^{(\{1\},\{2,3,4\})}=\frac{\beta^{2} q_{2} q_{3}\left(q_{3}-q_{2}\right)}{\left(4 q_{3}-q_{2}\right)^{2}}+\frac{1}{4} \frac{\beta^{2}\left(4 q_{2} q_{4}-q_{1} q_{4}-3 q_{1} q_{2}\right)}{\left(4 q_{2}-q_{1}\right)},
$$

while firm 1 obtains

$$
v(1 ;\{1,234\})=\Pi_{1}^{\{1,234\}}=\frac{\beta^{2} q_{1} q_{2}\left(q_{2}-q_{1}\right)}{\left(4 q_{2}-q_{1}\right)^{2}} .
$$


Note that, for $\beta=1, q_{1}=1, q_{2}=5$ and $q_{4}=10$ and $q_{3}>7.26$, the quality gap between $q_{2}$ and $q_{3}$ (both produced inside the cartel) becomes sufficiently high for

$$
\Pi_{1}^{\{1,234\}}+\Pi_{234}^{\{1,234\}}>\Pi_{N}^{\{N\}}=v(N)=\frac{1}{4} \beta^{2} q_{4}=2.5
$$

and the core is, as a result, empty. If, instead, products are equispaced, with $q_{1}=2.5$, $q_{2}=5, q_{3}=7.5$ and $q_{4}=10$,

$$
\Pi_{1}^{\{1,234\}}+\Pi_{234}^{\{1,234\}}=2.21<\Pi_{N}^{\{N\}}
$$

and also the other deviations by single or coalitions of firms cannot in any way improve upon the grand coalition payoff. Core existence is, in such a way, re-established.

\section{Concluding Remarks}

In this paper we have shown that in a vertically differentiated market when the variants marketed by the firms are equispaced, a fully collusive agreement in prices is core-stable. When this regularity condition is relaxed, the core can be easily empty.

\section{REFERENCES}

[1] Bloch, F, van den Nouweland, A., (2014) "Expectation Formation Rules and the Core of Partition Function Games", Games and Economic Behavior, 88, 339-353.

[2] Currarini, S., Marini, M. A. (2015) "Coalitional Approaches to Collusive Agreements in Oligopoly Games", The Manchester School, 83, 3, 253-287.

[3] de Clippel, G., Serrano, R. (2008), "Marginal Contributions and Externalities in the Value", Econometrica, 76, 6, 1413-1436.

[4] Hafalir, I. E. (2007) "Efficiency in Coalition Games with Externalities", Games and Economic Behaviour, 61, 242-258.

[5] Hart, S., Kurz, M. (1983), "Endogenous Formation of Coalitions", Econometrica, 52, 1047-1064.

[6] Friedman, J.W. (1989), Game Theory with Applications to Economics. Oxford University Press, Oxford.

[7] Gabszewicz, J. J., Thisse J-F. (1979) "Price Competition, Quality and Income Disparities" Journal of Economic Theory, 20, 340-359.

[8] Gabszewicz, J. J., Thisse J-F. (1980) "Entry (and Exit) in a Differentiated Industry", Journal of Economic Theory, 22, 327-338.

[9] Gabszewicz J.J., Marini, M. A. and Tarola, O. (2015), "Alliance Formation in a Vertically Differentiated Market", CORE Discussion Papers 30/2015, Université Catholique de Louvain, Louvain-la-Neuve, Belgium.

[10] Gabszewicz J.J., Marini, M. A. and Tarola, O. (2016), "Vertical Differentiation and Collusion: Proliferation or Cannibalization?", Research in Economics, forthcoming.

[11] Marini, M. A. (2016), "Collusive Agreements in Vertically Differentiated Markets", in Corchon, L. and Marini, M. A. (eds.), Handbook of Game Theory and Industrial Organization. Edward Elgar, Cheltenham and Northampton, Mass., forthcoming.

[12] Marini, M. A. (2009), "Games of Coalition and Network Formation: a Survey", in Naizmada A. et al. (eds), Networks, Dynamics and Topology, London \& Berlin, Springer-Verlag.

[13] Maskin E. (2003) "Bargaining, Coalitions and Externalities", Presidential Address to the Econometric Society, Institute for Advanced Study, Princeton University.

[14] Mussa, M., Rosen, S. (1978). Monopoly and product quality, Journal of Economic Theory, 18, 301-317.

[15] Ray, D. (2007). A Game-Theoretic Perspective on Coalition Formation. Oxford, U.K.: Oxford University Press.

[16] Ray D, Vohra, R. (2015) "Coalition Formation", Handbook of Game Theory with Economic Applications, Young, P. H., Zamir, S. (eds.), vol. 4, chapt. 5, 239-326, Elsevier.

[17] Thrall R., Lucas, W. (1963), "N-person Games in Partition Function Form", Naval Research Logistics Quarterly, 10, 281-298.

[18] Vives, X. (2000), Oligopoly Pricing. Old Ideas and New Tools. MIT Press, Cambridge, Mass. 
[19] Watanabe, T., Matsubayashi, N. (2013), "Note on Stable Mergers in Markets with Asymmetric Substitutability", Economics Bulletin, 33, 2024-2033.

[20] Zhao J. (2013) "The Most Reasonable Solution for an Asymmetric Three-firm Oligopoly", mimeo, Saskatchewan, Canada, March 2013. 